\title{
CONTINUOUS FUNCTIONS ON POLAR SETS
}

\author{
RAMASAMY JESURAJ
}

\begin{abstract}
Let $\Omega$ be a second countable Brelot harmonic space with a positive potential. If $K$ is a compact subset of $\Omega$ with more than one point, then $K$ is a polar set iff every positive continuous function on $K$ can be extended to a continuous potential on $\Omega$. This is a generalization of the result proved by $\mathrm{H}$. Wallin for the special case $\Omega=\mathbf{R}^{n}(n \geqslant 3)$ with Laplace harmonic space.
\end{abstract}

The objective of this paper is to generalize to axiomatic spaces of harmonic functions a well-known result of $\mathrm{H}$. Wallin [6] which states that a compact set $K \subset \mathbf{R}^{n}, n \geqslant 3$, has Newtonian capacity 0 iff every positive continuous function on $K$ is the restriction to $K$ of a positive Newtonian potential on the whole space. We give the generalization of this result at the setting of Brelot space [3], which contains a positive potential, and the topology of the space has countable space for open sets. Accordingly, we prove

THEOREM 1. Let $K$ be a compact subset contained in $\Omega$, a Brelot space as above, such that every positive continuous function on $K$ can be uniformly approximated on $K$ by positive superharmonic functions on $\Omega$. Further, let $K$ contain at least two points. Then $K$ is polar.

THEOREM 2. Let $K$ be a compact polar subset of $\Omega, f_{0}$ a positive continuous function on $K$, and $F_{0}$ a relatively compact open neighborhood of $K$. Then there is a continuous potential $p$ on $\Omega$ such that $p=f_{0}$ on $K$ and $p$ is harmonic on $\Omega \backslash \bar{F}_{0}$.

In view of the fact that there are Brelot spaces of the nature mentioned here in which point sets need not be polar [2], the restriction in Theorem 1 is quite warranted. We can write $K=F \cup G$, with $F$ and $G$ closed subsets of $\Omega$, neither of which is a subset of the other. Let $z$ be in $G$ and not in $F$. Then, by the Tietze extension theorem, for each positive integer $n$ there is a continuous function $f_{n}$ on $K$ such that $f_{n}>0$ on $K$, and $f_{n}(x)=1$ for all $x$ in $F$ and $f_{n}(z)=1 / 2^{n}$. Choose $\varepsilon$ such that $0<\varepsilon<1 / 2$. Then, by hypothesis, there is a positive superharmonic function $q_{n}$ on $\Omega$ such that $\left|f_{n}(x)-q_{n}(x)\right|<\varepsilon / 2^{n-1}$, i.e.,

$$
1-\frac{\varepsilon}{2^{n-1}}<q_{n}(x)<1+\frac{\varepsilon}{2^{n-1}} \text { for all } x \text { in } F,
$$

Received by the editors August 9, 1983.

1980 Mathematics Subject Classification. Primary 31D05.

(C)1985 American Mathematical Society $0002-9939 / 85 \$ 1.00+\$ .25$ per page 
and

$$
\frac{1}{2^{n}}-\frac{\varepsilon}{2^{n-1}}<q_{n}(z)<\frac{1}{2^{n}}+\frac{\varepsilon}{2^{n-1}} .
$$

Put $q(x)=\sum_{n=1}^{\infty} q_{n}(x)$ on $\Omega$. Then, obviously, $q$ is a superharmonic function and $q=\infty$ on $F$. Hence, $F$ is a polar set. Similarly, $G$ is also a polar set. Thus, $K$ is a polar set.

The proof of Theorem 2 relies on first providing an approximation which is in line with the conclusion of the Theorem 1 . We also include the following application, which is a generalization of another result of $\mathrm{H}$. Wallin (see [6, Remark 4, p. 62]).

THEOREM 3. Let $\Omega$ be a selfadjoint Brelot space [5] with second axiom of countability, $\delta$ a regular domain in $\Omega$, and $K$ a compact polar set $\subset \partial \delta$. Assume that the constant function 1 is superharmonic on $\Omega$. Then, given any positive continuous function $f$ on $K$, there is a continuous potential $p$ on $\Omega$ such that $p$ is harmonic on $\delta, f=p$ on $K$, and $D_{p}(\delta)<\infty$, where $D_{p}$ is the gradient measure, in the sense of Maeda, associated with $p$ [5].

Proof. By Theorem 2 there is a continuous potential $q_{1}$ on $\Omega$ such that $f=q_{1}$ on $K$. Since $f$ is continuous on $K$, there is a constant $b$ such that $0<f<b$ on $K$. Put $q=\min \left(q_{1}, b\right)$ on $\Omega$. Then $q$ is a bounded continuous potential on $\Omega$ and $q=f$ on $K$. Let

$$
p(x)= \begin{cases}\int q(z) d \rho_{x}^{\delta}(z) & \text { if } x \in \delta \\ q(x) & \text { if } x \in \Omega \backslash \delta .\end{cases}
$$

Then $p$ is a bounded continuous potential on $\Omega$, and $p$ is harmonic on $\delta$. By a result of Maeda [5], $D_{p}(\delta)<\infty$. Further, since $K \subset \partial \delta, f(x)=q(x)=p(x)$ for every $x$ in $K$. The proof is complete.

ACKNOWLEDGEMENT. The results of this paper formed part of a thesis submitted to McGill University. I would like to thank Professor K. N. Gowrisankaran for guiding my thesis and for his help in preparing this article.

1. Preliminaries. From now on, let $K$ denote a compact polar set in a Brelot space $\Omega$. The next theorem is a converse of Theorem 1 .

THEOREM 4. Given a positive continuous function on $K$ and an $\varepsilon>0$, there exists $a$ continuous potential $p$ on $\Omega$ such that $|f-p|<\varepsilon$ on $K$.

Proof. Let $\left\{U_{n}\right\}_{n=1}^{\infty}$ be a decreasing sequence of relatively compact open subsets of $\Omega$ such that $U_{n} \supset \bar{U}_{n+1} \supset U_{n+1}$ for $n=1,2,3, \ldots$, and $\cap_{n=1}^{\infty} U_{n}=K$. For each $n$, let $f_{n}$ be a continuous extension of $f$ to $\Omega$ such that $f_{n} \geqslant 0$ on $\Omega$, and $f_{n}$ has support contained in $U_{n}$. Taking the infimum at each stage, we may assume that $\left\{f_{n}\right\}_{n=1}^{\infty}$ is a decreasing sequence. Note that $f_{n} \rightarrow 0$ outside $K$ and $f_{n} \rightarrow f$ on $K$. From the fact that $\left\{R_{f_{n}}^{U_{n}}\right\}_{n=1}^{\infty}=\left\{R f_{n}\right\}_{n=1}^{\infty}$ is a decreasing sequence, it can be proved that $R f_{n} \rightarrow R_{f}^{K}$ pointwise on $\Omega$. However, it is routine to prove that $R_{f}^{K}=f$ on $K$. Hence, $R f_{n} \rightarrow f$ on 
$K$. Since, for each $n, R f_{n}$ is a continuous potential on $\Omega$ [2], by Dini's theorem, the convergence is uniform on $K$. Thus, there is an $m$ such that $\left|R f_{n}-f\right|<\varepsilon$ on $K$ if $n \geqslant m$.

THEOREM 5. Let $f_{0}$ be a positive continuous function on $K$, and let $F_{0}$ be a relatively compact open neighbourhood of $K$. Put $F=\bar{F}_{0}$ and let $f$ be a nonnegative continuous extension of $f_{0}$ to $\Omega$, such that $f>0$ on $F$. Then, given $\varepsilon>0$, there is a continuous potential $p$ on $\Omega$ such that $p<f$ on $F$ and $p \geqslant f_{0}-\varepsilon$ on $K$. In fact, $p$ can be chosen to be harmonic outside $F$.

Proof. We can assume $\varepsilon>0$ is small enough so that $f_{0}-\varepsilon>0$ on $K$. Apply the proof of the previous theorem to the function $f_{0}-\varepsilon$. Let $U_{n}$ be a sequence of relatively compact open subsets of $\Omega$ such that $U_{n} \supset \bar{U}_{n+1} \supset U_{n+1}, n=1,2,3, \ldots$ Let $g_{n}$ be a nonnegative, continuous extension of $f_{0}-\varepsilon$ to $\Omega$, with the support of $g_{n} \subset U_{n}$, and such that $R g_{n}$ converges to $\left(f_{0}-\varepsilon\right) \chi_{K}$ on $\Omega$ as $n \rightarrow \infty$.

Now, let $\eta>0$ such that $\eta<\varepsilon$ and $f_{0}-\varepsilon+\eta<f_{0}$ on $K$. Since $R g_{n}$ converges to $f_{0}-\varepsilon$ uniformly on $K$, there is an integer $n_{1}$ such that $R g_{n}<f_{0}-\varepsilon+\eta$ on $K$ if $n \geqslant n_{1}$. Since $R g_{n}$ and $f-\varepsilon+\eta$ are two continuous functions on $\Omega$, the set $V$, defined as $\left\{x\right.$ in $\left.\Omega: R g_{n}(x)<f(x)-\varepsilon+\eta\right\}$, is an open set containing $K$. Further, $R g_{n}$ is a decreasing sequence of functions on $\Omega$. Hence,

$$
R g_{n}(x)<f(x)-\varepsilon+\eta \text { for every } x \text { in } V \text { and every } n \geqslant n_{1} \text {. }
$$

Now $F_{0} \cap V$ is an open set which clearly contains $K$. This implies that there is an integer $n_{2}$ such that

$$
\bar{U}_{n} \subset V \cap F \text { if } n \geqslant n_{2} .
$$

Put $n_{3}=\max \left(n_{1}, n_{2}\right)$. Choose $\alpha>0$ such that $f \geqslant \alpha$ on $F$. Observe that $F \backslash U_{n_{3}}$ is a compact subset of $\Omega$, and $R g_{n}$ decreases to zero on $F \backslash U_{n_{3}}$ as $n \rightarrow \infty$. Hence, by Dini's theorem, the convergence is uniform, i.e., there is an integer $n_{4}$ such that $R g_{n} \leqslant \alpha / 2$ on $F \backslash U_{n}$ if $n \geqslant n_{4}$.

Put $m=\max \left(n_{3}, n_{4}\right)$ and $p=R g_{m}$. Then it follows that $p$ is a continuous potential; on $F \backslash U_{n_{3}}$,

$$
p=R g_{m} \leqslant R g_{n_{4}}<\alpha / 2<f
$$

and, lastly, using (1) and (2),

$$
p=R g_{m} \leqslant R g_{n_{3}} \leqslant R g_{n_{1}} \leqslant f-\varepsilon+\eta<f \text { on } U_{n_{3}} .
$$

Thus $p<f$ on $F$. Further, on $K, f_{0}-\varepsilon=g_{m} \leqslant R g_{m}=p$. The proof is complete.

2. Proof of Theorem 2. Now we prove the main theorem.

Put $F=\bar{F}_{0}$. Choose $f$, a nonnegative continuous extension of $f_{0}$ to $\Omega$, such that $f>0$ on $F$ and $f$ is zero outside a compact subset of $\Omega$. Let $\left\{U_{n}\right\}_{n=0}^{\infty}$ be a sequence of relatively compact open subsets of $\Omega$ such that $U_{n+1} \subset \bar{U}_{n+1} \subset U_{n}$ for $n=0,1,2, \ldots$, and $K=\bigcap_{n=0}^{\infty} U_{n}$.

We can assume that $U_{0} \subset F_{0}$. Fix a small $\varepsilon>0$.

By the previous theorem there is a continuous potential $p_{0}$ on $\Omega$ such that: (i) $p_{0}(x)<f(x)$ for every $x$ in $F$, (ii) $p_{0}(x) \geqslant f_{0}(x)-\varepsilon$ for every $x$ in $K$, and (iii) $p_{0}$ 
is a harmonic function on the open set $\Omega \backslash \bar{U}_{0}$. Let $f_{1}=\max \left(f-p_{0}, 0\right)$ on $\Omega$. Then $f_{1}$ is a continuous function on $\Omega$ and $f_{1}>0$ on $F$. Applying the previous theorem to $f_{1}$ and then proceeding inductively, we obtain a sequence $\left\{p_{n}\right\}_{n=1}^{\infty}$ of continuous potentials on $\Omega$ such that: (i) $p_{i}$ is a harmonic function on the open set $\Omega \backslash \bar{U}_{i}$ for every $i$, (ii) $\sum_{i=0}^{n} p_{i}<f$ on $F$ for every $n$, and (iii) $\sum_{i=0}^{n} p_{i} \geqslant f_{n}-\varepsilon / 2^{n}$ on $K$ for every $n$.

Put $p=\sum_{i=0}^{\infty} p_{i}$. It is clear that $p$ is a potential function on $\Omega$. Further, on $K$,

$$
f_{0}-\varepsilon / 2^{n} \leqslant \sum_{i=0}^{n} p_{i}<f=f_{0} \text { for every } n .
$$

Taking the limit as $n \rightarrow \infty$, we conclude that $p=f_{0}$ on $K$.

Now $p_{i}$ is a harmonic function outside $\bar{U}_{i} \subset F_{0} \subset F$ for every $i$. Hence, $\sum_{i=0}^{n} p_{i}$ is a harmonic function outside $F$. Therefore, on every connected component of $\Omega \backslash F$, $p=\lim _{n \rightarrow \infty} \sum_{i=0}^{n} p_{i}$ is either a harmonic function or identically $+\infty$. But $p$ is a superharmonic function on $\Omega$. This implies that $p$ is finite on a dense subset of $\Omega$, which eliminates the second possibility. Hence, $p$ is a harmonic function on $\Omega \backslash F$. Thus, we are left with proving the continuity of $p$ on $\Omega$.

Let $z$ be in $\Omega \backslash F$. Since $p$ is a harmonic function on the open set $\Omega \backslash F$, it is continuous at $z$. Let $z$ be in $K$. Then

$$
\begin{aligned}
p(z) & \leqslant \liminf _{x \rightarrow z} p(x) & & (\text { by lower semicontinuity of } p) \\
& \leqslant \limsup _{x \rightarrow z} p(x) & & \\
& =\limsup _{x \rightarrow z x \in F_{0}} p(z) & & \left(\text { as } F_{0} \text { is an open neighbourhood of } z\right) \\
& \leqslant f(z) & & (\text { as } f \text { is continuous at } z) \\
& =f_{0}(z) & & \\
& =p(z) & & \left(\text { as } p=f_{0} \text { on } K\right) .
\end{aligned}
$$

Hence, $p$ is continuous at $z$.

Now, let $z$ in $F \backslash K$. Then there exists an integer $N$ such that $z \notin U_{n}$ if $n \geqslant N$. By writing $p(x)=\sum_{i=0}^{N-1} p_{i}(x)+\sum_{i=N}^{\infty} p_{i}(x)$, we see that $\sum_{i=N}^{\infty} p_{i}(x)$ is a harmonic function in an open neighbourhood of $z$. In particular, $\sum_{i=N}^{\infty} p_{i}(x)$ is continuous at $z$. Being a finite sum of continuous functions at $z, \sum_{i=0}^{N-1} p_{i}$ is a continuous function at $z$. Hence, $p$ is continuous at $z$. Thus, $p$ is continuous on $\Omega$, and the proof is complete.

Corollary 6. Assume that the constant functions are harmonic on $\Omega$. If $K$ is $a$ compact polar subset of $\Omega$, then every continuous function on $K$ can be extended to $a$ superharmonic function on $\Omega$.

\section{BIBLIOGRAPHY}

1. M. Brelot, Lectures on potential theory, Tata Inst. Fund. Res., Bombay, 1960; reissued 1967.

2. C. Constantinescu and A. Cornea, Potential theory on harmonic spaces, Springer-Verlag, 1972.

3. R. M. Herve, Recherches axiomatiques sur la theorie de fonctions surharmoniques et du potential, Ann. Inst. Fourier (Grenoble) 12 (1962), 415-571. 
4. R. Jesuraj, Continuous functions and exceptional sets in potential theory, Ph.D. Thesis, McGill University, Montreal, 1981.

5. F. Y. Maeda, Dirichlet integrals on harmonic spaces, Lecture Notes in Math., vol. 803, Springer-Verlag.

6. H. Wallin, Continuous functions and potential theory, Ark. Mat. 5 (1963), 55-84.

Department of Mathematics and Computer Science, State University of New York, BrockPORT, NEW YORK 14420

Current address: Prime Computer, Incorporation, 500 Old Connecticut Path, Framingham, Massachusetts 01701 